\title{
Study of Relationship Between Dry Eye and Expressions of CXCR3 and CCR5 After Ocular Acid Burn
}

\section{Bo Jiang}

Jinshan Hospital affiliated to Fudan University

Qianqian Hu

Huadong Hospital affiliated to Fudan University

Tao Li

Jinshan Hospital affiliated to Fudan University

Man She

Jinshan Hospital affiliated to Fudan University

Xiaodong Zhou (Dxdzhou2013@126.com)

Jinshan Hospital affiliated to Fudan University

\section{Research Article}

Keywords: Burn, acid, ocular, CXCR3, CCR5

Posted Date: November 30th, 2021

DOI: https://doi.org/10.21203/rs.3.rs-1119696/v1

License: (c) (i) This work is licensed under a Creative Commons Attribution 4.0 International License. Read Full License 


\section{Abstract \\ Objective}

To investigate the manifestation of dry eye and its relationship with CXCR3 and CCR5 expression in patients with ocular acid burns.

\section{Methods}

This is a case-control study. A total of 27 eyes of 22 cases ocular with acid burns of I-V degrees from Jan.2020 to Feb.2021 in this hospital were selected as observation group, and 8 eyes of 8 cases of normal people were selected as control group. The followed up time was 3 months. The visual acuity, intraocular pressure, BUT, Schirmer $\otimes$ test, corneal thickness and tear meniscus height (TMH) were observed at 1 day, 1 and 3 months after injury. The protein expression of CXCR3 and CCR5 were examined by ELISA and were compared among groups at each time point.

\section{Results}

BUT and Schirmer I tests value in the observation group were lower than those in the control group 3 months after injury (all $p<0.05$ ). The corneal thickness and the tear meniscus height 1 day after injury were higher than those in the control group $(p<0.05)$, and also higher than those at 1 month and 3 months after injury $(p<0.05)$. The expression levels of CXCR3 and CCR5 protein were significantly negatively correlated with BUT $(p<0.05)$, and CXCR3 and CCR5 were also significantly negatively correlated with Schirmer I test value $(p<0.05)$.

\section{Conclusion}

Ocular acid burns can cause dry eye, and the expression of CXCR3 and CCR5 protein in tears may be related to the occurrence of dry eye after ocular acid burn.

\section{Background}

Ocular chemical burn, including acid burn and alkali burn, is one of the common eye injuries, accounting for $22 \%$ of ocular trauma[1], with acute onset and rapid progression, often leading to a variety of complications. And dry eye is one of the common complications of acid burn. Therefore, the diagnosis and preventive treatment of dry eye after ocular acid burn have become the focus of attention. Studies have shown that dry eye is a multi-factor ocular disease characterized by the imbalance of tear film homeostasis and accompanied by uncomfortable symptoms[2]. In the studies of various types of dry eye disease, the increase of ocular surface inflammatory factors and chemokines has been found to induce immune inflammatory response, which may be an important co-pathogenesis of dry eye disease[3, 4, 5]. 
Among them, chemokine receptors CXCR3 (CXC chemokine receptor-3, CXCR3) and CCR5 (CC chemokine receptor-5, CCR5) act as surface markers of activated Th1 lymphocytes to mediate ocular surface inflammation[6]. Therefore, it may be of clinical significance to investigate the relationship between dry eye and expression of CXCR3 and CCR5 after ocular acid burn. In this study, the regularity of dry eye manifestations after acid burn and its relationship with CXCR3 and CCR5 were studied, providing clinical reference for the diagnosis and treatment of dry eye after acid burn.

\section{Methods}

\subsection{Patients}

This study collected data from twenty-two patients with a primary diagnosis of ocular acid burn in the Department of Ophthalmology, Jinshan Hospital affiliated to Fudan University, China, from January 2020 to February 2021. All patients were diagnosed according to the International Classification of Diseases, Tenth Revision, Clinical Modification (ICD-10-CM). This study was approved by the Ethics Committee of Jinshan Hospital of Fudan University (IEC-2020-S03). All procedures conformed to the tenets of the Declaration of Helsinki. Written informed consent was obtained from all patients.

\subsubsection{Inclusion criteria}

(1) The patient was definitely diagnosed with acid ocular burn. (2) The patient had no history of dry eye, contact lens wearing, or eye surgery. (3) The patient didn't have glaucoma, chronic dacryocystitis and eye diseases. (4) Patient was excluded from sjogren's syndrome, connective tissue disease, diabetes and other related diseases.

\subsubsection{Clinical data}

Twenty-two patients (27 eyes) with acid ocular burn treated in our hospital from January 2020 to February 2021 were selected as the observation group, including 18 males (22 eyes) and 4 females (5 eyes), ranging in age from 28 to 62 years, with an average age of $(44.0 \pm 9.6)$ years. The time of visit was 30 minutes to 24 hours after injury, and all patients had their eyeballs irrigated with water at the scene. The degree of burn was in accordance with GBZ 54-2017 diagnostic criteria for occupational chemical eye burn[7], including 8 patients with grade I, grade II and grade III chemical burn, 2 patients with grade IV chemical burn, and 1 patient with grade $V$ chemical burn. According to the degree of burn, the patients with grade I burn were divided into group I including 8 eyes, the patients with grade II burn were divided into group II including 8 eyes, the patients with grade III burn were divided into a group III including 8 eyes, and the patients with grade IV and grade $\mathrm{V}$ burn were divided into group IV including 3 eyes.

At the same time, 8 normal people without dry eyes (8 eyes) were selected as the control group, including 5 males and 3 females, aged 28-56 years, with an average age of $(40.5 \pm 9.0)$ years, and there was no significant difference in age from the observation group $(P<0.05)$.

\subsection{Methods}




\subsubsection{Treatment}

The conjunctival sac of all injured patients was thoroughly rinsed with normal saline immediately after injury in the hospital, followed by topical antibiotic eye drops, eye ointment (levofloxacin eye drops, levofloxacin eye ointment) and eye drops that promote corneal repair (recombinant bovine basic fibroblast growth factor eye drops). Local glucocorticoid eye drops (tobramycin and dexamethasone eye drops) were administered within 1 week after injury, and amniotic membrane transplantation was performed in patients with grade III or higher burns.

\subsubsection{Observation Indicators}

Visual acuity, intraocular pressure (IOP), tear meniscus height (TMH), corneal thickness, breakup time of tear film (BUT) and Schirmer I tests were performed in the control group and observation group at 1 day, 1 month and 3 months after injury, respectively. Meanwhile, the protein expression levels of CXCR3 and CCR5 were determined by Elisa.

(1) Breakup time of tear film (BUT): $1 \%$ fluorescein sodium was dropped into the conjunctiva sac, and slit lamp observation was performed after several blinks. BUT was the time from eye opening after the last blink to the first black spot on the cornea. Average value was taken for three consecutive measurements, normal BUT was $\geq 10$ seconds.

(2) Schirmer I test: The Schirmer filter paper was placed in the conjunctival sac of the subject under normal indoor light, and removed after 5 minutes to measure the wetting length, which was the value of Schirmer I test. The wetting length of the filter paper was $\geq 10 \mathrm{~mm}$ after 5 minutes in normal subjects.

(3) Measurement of tear meniscus height (TMH) and corneal thickness: Cirrus-HD 4000 OCT (optical coherent tomography, Zeiss, Germany) was adopted. The light source was near infrared light at $1310 \mathrm{~nm}$. The scanning range was $10 \mathrm{~mm}$ in transverse direction and $3 \mathrm{~mm}$ in longitudinal direction, with resolutions of 19 um and 4 um respectively. Adopt the single-line scanning mode of internal fixation device to adjust the fixation Angle so that the optic axis is consistent with the optical axis. The high resolution mode was selected, and the scan line was adjusted to the tear meniscus below the vertical cornea center. When the screen showed a high reflective light that marked the cornea center, the subject was instructed to blink, and the image was acquired immediately after the image stabilized. Scan continuously for 3 times, measure the lower $\mathrm{TMH}$ with its own software caliper tool, and take its average value. After the TMH was measured, the central thickness of the cornea was measured with the OCT builtin program. During the examination, the patient was instructed to gaze at the red light in front, and the corneal light band was adjusted between the target band. The scan line length was set to $3 \mathrm{~mm}$, and the horizontal scan was performed, and the image was captured and saved.

(4) Collection and determination of CXCR3 and CCR5: 10ul of the patient's tears were absorbed by a disposable capillary tear collector and placed in a $0.5 \mathrm{~mL}$ EP tube, stored at $-80^{\circ} \mathrm{C}$. The protein expressions of CXCR3 and CCR5 were detected by Elisa. 


\subsection{Statistical Analysis}

SPSS version 22.0 software was used for statistical analysis. Biologic parameter data of each group were pairwise compared, Levene test was used for homogeneity of variance test, Bonferroni was used for pairwise comparison when the variance was homogeneous, and Dunnett's T3 was used for pairwise comparison when the variance was inhomogeneous. The independent sample $T$ test was used to compare the Elisa gray values between the two groups, and Pearson correlation analysis was used to test the correlation between biological parameters and the expression of CXCR3 and CCR5. All statistical results are expressed as mean \pm standard error of the mean (SEM) and a p value $<0.05$ was considered statistically significant.

\section{Results}

\subsection{Comparison of visual acuity, IOP, BUT and of Schirmer I test value at 1 day, 1 month and 3 months after injury in each group.}

Visual acuity, IOP, BUT and Schirmer I tests of patients were performed in the observation group at 1 day, 1 month and 3 months after injury, respectively. The same tests were performed in the control group on the first day of enrollment. And the results were shown as follows (Table 1).

Visual acuity: Compared with the control group, the visual acuity of patients with ocular acid burn in group I-IV significantly decreased 1 day after injury (all $p<0.05$ ). 3 months after injury, there was no significant difference in visual acuity between patients in group I-III and those in the control group (all $p>$ $0.05)$. And the visual acuity of patients in group IV was still significantly lower than those in the control group 3 months after injury $(p<0.05)$. Compared with 1 day after injury in the same group, the visual acuity of patients with ocular acid burn in group I-III was significantly improved 3 months after injury (all $p<0.05)$ whereas the visual acuity of patients in group IV didn't significantly improve 3 months after injury $(p>0.05)$.

IOP: Compared with the control group, IOP of patients with ocular acid burn in group I-IV was significantly increased 1 day after injury (all $p<0.05$ ). Although IOP in group I-III increased, it was still within the normal range while the IOP in group IV exceeded the upper limit of normal value. There was no significant difference in IOP between IIV group and control group 3 months after injury (all $p>0.05$ ), indicating that IOP in the four groups returned to the daily level three months later. Compared with 1 day after the injury in the same group, IOP of patients with ocular acid burn in group II-IV decreased significantly 3 months after the injury (all $p<0.05$ ). However, there was no significant decrease in IOP of patients in group I, which may be due to the mild degree of injury and the difference in IOP between 3 months after recovery and 1 day after injury was too small to be statistically significant.

BUT: Compared with the control group, patients with ocular acid burn in groups I-IV had significantly shorter BUT 3 months after injury (all $p<0.05$ ). 1 day after injury, however, BUT in groups II and III was longer than that in the control group (both $p<0.05$ ) while BUT in groups I and IV was not significantly 
different from that in the control group (both $p>0.05$ ). These results suggest that there was no regular difference in BUT between group I-IV and the control group 1 day after acid burn injury, which may be due to the high degree of corneal edema 1 day after injury that affected the timing of tear film rupture. When compared with 1 day after injury in the same group, BUT was significantly shorter at 1 and 3 months after injury in the I-IV group (all $p<0.05$ ).

Schirmer I test: Compared with the control group, the values of Schirmer I test were significantly higher in patients with ocular acid burn in groups II-IV 1 day after injury; while the values of Schirmer I test significantly decreased in patients with ocular acid burn in groups I-IV 3 months after injury (all $p<0.05)$. When compared with 1 day after injury in the same group, Schirmer I test values of patients with ocular acid burn in the I-IV group were significantly shorter at 1 and 3 months after injury (all $p<0.05$ ).

Table 1. Comparison of biological parameters at different times in each group

\begin{tabular}{|c|c|c|c|c|c|}
\hline $\begin{array}{l}\text { Group } \\
\text { Parameter }\end{array}$ & & Visual acuity & IOP & BUT & Schirmer I test \\
\hline \multirow{3}{*}{$\begin{array}{l}\text { Group I } \\
\text { (Grade I) }\end{array}$} & 1 day & $0.70 \pm 0.06^{*}$ & $18.38 \pm 1.00^{\star}$ & $13.50 \pm 0.87$ & $13.75 \pm 0.98$ \\
\hline & 1 month & $0.85 \pm 0.05$ & $16.63 \pm 0.65$ & $10.00 \pm 0.76^{\#}$ & $8.50 \pm 0.42^{\#}$ \\
\hline & 3 month & $0.95 \pm 0.03^{\#}$ & $16.50 \pm 0.68$ & $8.75 \pm 0.70^{\star \#}$ & $9.25 \pm 0.37^{\star \#}$ \\
\hline \multirow{3}{*}{$\begin{array}{l}\text { Group II } \\
\text { (Grade II) }\end{array}$} & 1 day & $0.58 \pm 0.03^{*}$ & $19.25 \pm 0.88^{\star}$ & $15.00 \pm 0.76$ & $15.75 \pm 1.00^{*}$ \\
\hline & 1 month & $0.80 \pm 0.05$ & $16.50 \pm 0.82$ & $10.00 \pm 0.27^{\#}$ & $10.63 \pm 0.53^{\#}$ \\
\hline & 3 month & $0.88 \pm 0.05^{\#}$ & $16.00 \pm 0.82^{\#}$ & $8.75 \pm 0.65^{\star \#}$ & $8.88 \pm 0.61^{\star \#}$ \\
\hline \multirow{3}{*}{$\begin{array}{l}\text { Group III } \\
\text { (Grade III) }\end{array}$} & 1 day & $0.29 \pm 0.03^{*}$ & $19.88 \pm 0.58^{*}$ & $13.63 \pm 0.57$ & $18.88 \pm 0.72^{*}$ \\
\hline & 1 month & $0.65 \pm 0.07$ & $16.13 \pm 0.51$ & $8.50 \pm 0.63^{\#}$ & $11.13 \pm 0.30^{\#}$ \\
\hline & 3 month & $0.83 \pm 0.06^{\#}$ & $16.25 \pm 0.37^{\#}$ & $6.88 \pm 0.58^{\star \#}$ & $9.00 \pm 0.38^{\star \#}$ \\
\hline \multirow{3}{*}{$\begin{array}{l}\text { Group IV } \\
\text { (Grade IV \& V) }\end{array}$} & 1 day & $0.06 \pm 0.02^{*}$ & $22.33 \pm 1.45^{\star}$ & $14.00 \pm 1.16$ & $20.33 \pm 0.88^{*}$ \\
\hline & 1 month & $0.10 \pm 0.02^{*}$ & $17.67 \pm 1.76$ & $10.67 \pm 1.33^{\#}$ & $11.67 \pm 0.88^{\#}$ \\
\hline & 3 month & $0.12 \pm 0.01^{*}$ & $17.67 \pm 0.88^{\#}$ & $6.67 \pm 0.67^{\star \#}$ & $7.00 \pm 0.58^{\star \#}$ \\
\hline Control group & & $0.93 \pm 0.04$ & $15.88 \pm 0.52$ & $11.25 \pm 0.73$ & $12.38 \pm 0.78$ \\
\hline
\end{tabular}

Note: a. Compared with the control group, ${ }^{*} p<0.05$; b. Compared with 1 day after injury in the same group, $\#_{p}<0.05$.

3.2 Comparison of corneal thickness at 1 day, 1 month and 3 months after injury in each group. 
The comparison of corneal thickness at different time points in each group and OCT images of corneal thickness of a patient in group III were shown in Figure 1. The corneal thickness of the control group was $(537.00 \pm 2.59) \mu \mathrm{m} .1$ day after injury, the corneal thickness of patients with ocular acid burn in the I-IV group was $(540.50 \pm 4.50) \mu \mathrm{m},(553.75 \pm 3.10) \mu \mathrm{m},(571.00 \pm 3.36) \mu \mathrm{m},(605.33 \pm 8.11) \mu \mathrm{m}$, respectively. The corneal thickness of patients with ocular acid burn in the II-IV group 1 day after injury was significantly thicker than that in the control group (all $p<0.05$ ).

1 month after injury, the corneal thickness of patients with ocular acid burn in the I-IV group were $(530.50 \pm 4.12) \mu \mathrm{m},(544.50 \pm 2.44) \mu \mathrm{m},(553.00 \pm 2.10) \mu \mathrm{m},(564.00 \pm 4.62) \mu \mathrm{m}$, respectively. The corneal thickness of patients with ocular acid burn in II-IV group at 1 month after injury was significantly thinner than that at 1 day after injury (all $p<0.05$ ).

3 months after injury, the corneal thickness of patients with ocular acid burn in the II-IV group were $(529.50 \pm 4.21) \mu \mathrm{m},(540.25 \pm 2.52) \mu \mathrm{m},(546.00 \pm 2.51) \mu \mathrm{m},(541.33 \pm 3.53) \mu \mathrm{m}$, respectively. The corneal thickness of patients with ocular acid burn in II-IV group 3 months after injury was significantly thinner than that 1 day after injury (all $p<0.05$ ).

\subsection{Comparison of tear meniscus height (TMH) at 1 day, 1 month and 3 months after injury in each group.}

The comparison of tear meniscus height (TMH) at different time points in each group and OCT images of corneal thickness of a patient in group III were shown in Figure 2. The TMH of the control group was (228.00 \pm 6.93$) \mu \mathrm{m} .1$ day after injury, the TMH of patients with ocular acid burn in the I-IV group was $(241.25 \pm 7.70) \mu \mathrm{m},(268.50 \pm 4.31) \mu \mathrm{m},(313.50 \pm 11.90) \mu \mathrm{m},(552.00 \pm 77.25) \mu \mathrm{m}$, respectively. 1 month after injury, the TMH of patients with ocular acid burn in the I-IV group were $(216.00 \pm 5.24) \mu \mathrm{m},(217.00 \pm 6.67)$ $\mu \mathrm{m},(198.50 \pm 6.97) \mu \mathrm{m},(214.67 \pm 16.38) \mu \mathrm{m}$, respectively. 3 months after injury, the TMH of patients with ocular acid burn in II-IV group were $(199.00 \pm 5.39) \mu \mathrm{m},(199.50 \pm 8.80) \mu \mathrm{m},(188.50 \pm 7.61) \mu \mathrm{m}$, $(176.00 \pm 6.11) \mu \mathrm{m}$, respectively. Compared with the control group, the TMH of patients with ocular acid burn in II-IV group significantly increased 1 day after injury (all $p<0.05)$, and patients with ocular acid burn in I-IV group had significantly lower TMH 3 months after injury (all $p<0.05$ ). When comparing the TMH of patients with ocular acid burn in the same group, the TMH of patients in the I-IV group at 1 month after injury was significantly lower than that at 1 day after injury, and the TMH of patients in the I-IV group at 3 months after injury was significantly lower than that at 1 month after injury (all $p<0.05$ ). (Figure 2 )

\subsection{Expression of CXCR3 and CCR5 of patients in each group at 1 day, 1 month and 3 months after injury.}

The protein expression levels of CXC-chemokine receptor 3 (CXCR3) and CC-chemokine receptor 5 (CCR5) in tears of patients with ocular acid burn in each observation group were detected by ELISA at 1 day, 1 month and 3 months after injury. And the protein expression levels of CXCR3 and CCR5 in tears of patients in the control group were detected after they were recruited. The results are as follows. (figure 3 ) 
The protein levels of CXCR3 in tears of patients in each group were compared at different times after injury. Compared with the control group, CXCR3 protein level in tears of patients with ocular acid burn in group II-IV was significantly increased 3 months after injury (all $p<0.05$ ), and no significant change was found in the protein level of CXCR3 in tears of patients in group I 3 months after injury $(p>0.05)$. There was no significant difference in protein levels of CXCR3 in tears between the group I-IV and the control group 1 day and 1 month after injury (all $p>0.05$ ). When compared with 1 day after injury in the same group, the protein levels of CXCR3 in tears of patients with ocular acid burn in group II-IV significantly increased 3 months after injury, but no significant change was observed in group I 3 months after injury $(p>0.05)$. And there was no significant difference in protein levels of CXCR3 of tears in group I-IV between 1 day and 1 month after injury (all $p>0.05$ ). (figure $3 A$ )

The protein levels of CCR5 in tears of patients in each group were compared at different times after injury. The protein levels of CCR5 in tears of patients with ocular acid burn in group I-IV was significantly higher than that in the control group at 1 month and 3 months after injury (all $p<0.05$ ). The protein levels of CCR5 in tears of patients in group IV was significantly higher than that in the control group at 1 day after injury $(p>0.05)$. And there was no significant difference in the protein levels of CCR5 in tears between the group IIIV and the control group 1 day after injury (all $p>0.05$ ). When compared with 1 day after injury in the same group, the protein levels of CCR5 in tears of patients with ocular acid burn in the IIV group significantly increased 3 months after injury (all $p<0.05$ ), and no significant change was found in the protein level of CCR5 in tears of patients in group I-IV 1 month after injury (all $p>0.05$ ). (figure 3B)

\subsection{Correlation analysis of CXCR3 and CCR5 protein expression levels with ocular biological parameters.}

The protein expressions of CXCR3 and CCR5 in tears of acid burn patients in each group at different time points after injury were analyzed by Pearson correlation with their ocular biological parameters. The GraphPad Prism 8 software was used to make correlation images. The expression of CXCR3 in tears of patients with ocular acid burn was significantly negatively correlated with BUT $(R=-0.4710, P<0.0001$, Figure 4A), and the expression of CXCR3 in tears of patients with ocular acid burn was also significantly negatively correlated with values of Schirmer I test $(R=-0.4864, P<0.0001$, Figure $4 B)$. The expression of CCR5 in tears of patients with ocular acid burn was significantly negatively correlated with BUT $(R=-0.4173, P<0.0001$, Figure $4 C)$, the expression of CCR5 in tears of patients with ocular acid burn was also significantly negatively correlated with Schirmer I test $(R=-0.3650, P=0.0004$, Figure 4D), and CXCR3 and CCR5 in tears of patients with ocular acid burn were not significantly correlated with other indicators.

\section{Discussion}

Our data indicate that ocular acid burn causes significant damage to the structure and function of the eye, resulting in significant changes in ocular biological parameters. Specifically, the visual acuity of patients with ocular acid burn decreased significantly one day after injury, and the visual acuity of patients with severe acid burn (grade IV burn and above) was irreversibly damaged after injury, and their visual acuity could not return to normal 3 months after injury. Intraocular pressure (IOP) increased one 
day after ocular acid burn, but returned to normal 3 months after injury. The tear film break-up time (BUT) was significantly prolonged one day after ocular acid burn, but significantly shortened and lower than normal 3 months after injury. Similarly, Schirmern I test values increased significantly one day after ocular acid burn, but decreased significantly and were lower than normal 3 months after injury. Corneal thickness significantly increased one day after acid burn, but returned to normal 3 months after injury. The tear meniscus height (TMH) increased significantly one day after ocular acid burn, but decreased significantly and was lower than normal 3 months after ocular acid burn. In addition, the levels of CXCR3 and CCR5 in tears increased significantly one day after ocular acid burn, and returned to normal 3 months later. Notably, Pearson correlation analysis showed that CXCR3 and CCR5 expressions were significantly negatively correlated with BUT and Schirmer I test values. CXCR3 and CCR5 in tears significantly increased 1 day after injury, and the values of BUT and Schirmer I test were also significantly increased at this time. However, 3 months after injury, CXCR3 and CCR5 were significantly decreased, and BUT and Schirmer I test values were also significantly decreased. These results indicate that CXCR3 and CCR5 play an important role in the occurrence of dry eye after ocular acid burn.

Ocular acid burn is a kind of chemical eye injury, which can be divided into alkali burn and acid burn according to etiology. Although acid injuries tend to be less severe than alkali injuries, it can still lead to a range of sequelae, including potential corneal ulceration/perforation, secondary open angle glaucoma, corneal scarring, limbal stem cell deficiency, dry eye, etc[8]. Studies have shown that chemical ocular injuries (acid and alkali burns) can cause extensive damage leading to visual impairment by causing widespread inflammation, scarring, melting, and necrosis of the ocular structures[9, 10,11]. At the same time, severe periorbital edema or inflammation of the trabecular meshwork after a burn can lead to high intraocular pressure[9]. In our study, patients with various degrees of acid burn had visual impairment, increased IOP and corneal edema and thickening 1 day after injury, and the more severe the acid burn, the more severe the impairment of visual acuity and IOP, and the more severe the corneal edema and thickening. In addition, dry eye is a common complication after chemical eye injury, and its mechanism still remains unclear[12]. It has been suggested that due to the destruction of the meibomian gland in the tarsal conjunctiva, burn patients often lack lipid in tear and therefore are predisposed to develop evaporative dry eye[13]. And evaporative-type dry eye disease was found to have a close clinical correlation with tear cytokines and chemokines[14]. In addition, many studies have suggested that the mechanism of dry eye after chemical eye injury is related to the inflammation factors mediated inflammatory reaction, and blocking the inflammation factors mediated signaling pathway can reduce inflammation, thus decreasing the apoptosis of corneal epithelium in dry eye after chemical eye injury and improving wound healing[15, 16, 17]. Inflammatory factors involved in the immune inflammatory response of dry eye include cytokines, chemokines and soluble receptors, among which chemokines and their receptors are widely used in clinical and experimental studies of dry eye[18]. In this study, 3 months after injury, BUT of patients with ocular acid burn was significantly shortened, the Schirmer I test value was significantly reduced, and the tear meniscus height (TMH) was significantly reduced, indicating that ocular acid burn caused the long-term complication - dry eye. Notably, we detected the levels of CXCR3 and CCR5 in tears of patients with ocular acid burn, and analyzed their correlation with the biological 
parameters of the patients' eyeballs. We found a significant negative correlation between CXCR3 and CCR5 levels in tears of patients with ocular acid burn and values of BUT and Schirmer I tests. These results suggest that CXCR5 and CCR5 may be involved in the occurrence of dry eye after ocular acid burn.

C-X-C chemokine receptor 3 (CXCR3) and C-C chemokine receptor 5 (CCR5) are important classical chemokine receptors, which are cytokines that can cause immune inflammatory response by inducing lymphocyte migration through binding with ligands. Lymphocyte migration and phagocytosis are key mechanisms of immune inflammatory response, which is considered to be one of the important pathogenesis of dry eye[19], and CXCR3 and CCR5 play an important role in it[20,21]. The tear nature and compositions play a critical role in the pathogenesis of the dry eye. It is urgent to study the expression and change of inflammatory factors in tears for the prevention and treatment of dry eye disease. Similar to the previous reports, in this study, chemokine receptors CCR5 and CXCR3 were found expressed in the tears of patients with dry eye[22]. However, how CCR5 and CXCR3 functions on the development of dry eye and whether the content changes of CXCR3 and CCR5 in tears related to the disease progression of dry eye requires further studies.

Choi's study suggests that by blocking the chemokine receptors, CCR5 as well as CXCR3, and their specific ligands, it may be possible to modulate the manifestations of immunopathologic responses at the ocular surface in dry eye disease[23]. Zhengri's study reveals that topical application of infliximab can decline tear CXCR3 expression and improve the clinical and histological parameters[24]. Similarly, topical APN-derived short peptides (ADPs) could effectively decrease the production of inflammatory factors, such as CXCR3 and CCR5, and improve clinical signs of experimental eye dry or alkali burn[25]. Presented evidence suggests that CXCR3 and CCR5 are potential therapeutic targets for dry eye. Firstly, the extracellular ligand IL- 6 directs T cell recruitment by regulating local chemokine secretion and chemokine receptor (CCR4, CCR5, CXCR3, etc.) expression on the $\mathrm{CD}^{+}{ }^{+}$infiltrate, thus leading to the occurrence of dry eye[18]. Secondly, when the chemokine receptor CXCR3 (along with CCR5) expressed by Th1 cells binds to their specific ligands, such as INF- $\gamma$, they act as a central mediator to coordinate the localization of $\mathrm{CD} 4^{+} \mathrm{T}$ cells to the ocular surface to perform an immune response, causing dry eye occurrence[26]. Therefore, a reasonable assumption was that after ocular acid burn, the contents of inflammatory factors CXCR3 and CCR5 in the eyes of patients are increased, and their combination with ligand leads to recruitment of T1 lymphocytes on the ocular surface, which leads to immune inflammatory response on the ocular surface, thus forming the long-term complications of dry eye. Furthermore, when CXCR3 and CCR5 act as the virus cellular co-receptor, CXCR3 and CCR5 antagonists have been used in the treatment of HIV infection in clinical practice[27], indicating the modulation of the CXCR3 and CCR5 pathway might be a potential therapeutic strategy. In this study, CXCR3 and CCR5 in tears of patients with ocular acid burn were found to be closely related to the manifestations of dry eye, indicating that CXCR3 and CCR5 are involved in the occurrence of dry eye after ocular acid burn. Therefore, it deserves further exploration to reveal whether the regulation of CXCR3 and CCR5 pathways to control the immune inflammatory response can be used for the prevention and treatment of dry eye after ocular acid burn. 


\section{Conclusion}

This study discovered that ocular acid burn would not only damage visual acuity and intraocular pressure, but also lead to long-term complications of dry eye. Furthermore, dry eye after ocular acid burn is closely related to the expression of chemokine receptors CXCR3 and CCR5 in tears. CXCR3 and CCR5 may be potential targets for the prevention and treatment of dry eye after ocular acid burn. However, further studies investigating the mechanisms and functions of CXCR3 and CCR5 in dry eye after ocular acid burn are needed to explore better treatment.

\section{Abbreviations}

CXCR3: CXC chemokine receptor-3; CCR5: CC chemokine receptor-5; IOP: intraocular pressure; BUT: breakup time of tear film; TMH: tear meniscus height; ADPs: APN-derived short peptides; SEM: standard error of the mean.

\section{Declarations}

\section{Acknowledgments}

Authors thank patients for their kindly agreed to be included in the current study.

\section{Authors' contributions}

Bo Jiang and Tao Li performed the measurement of biological parameters. Man She performed the Molecular Biology experiment. Qainqian Hu analyzed the data and wrote the original manuscript. Xiaodong Zhou critically revised the manuscript during all submission process. All authors read and approved the final manuscript.

\section{Funding}

The study was supported by the Youth Fund Project of Jinshan Hospital affiliated to Fudan University (JYQN-LC-202008). The funder had no role in study design, data collection and analysis, decision to publish, or preparation of the manuscript.

\section{Availability of data and materials}

The datasets used and analyzed during the current study are available from the corresponding author on reasonable request.

\section{Ethics approval and consent to participate}

This study was approved by the Ethics Committee of Jinshan Hospital of Fudan University (IEC-2020S03). All procedures conformed to the tenets of the Declaration of Helsinki. Written informed consent was obtained from all patients. 


\section{Consent for publication}

Not applicable.

\section{Competing interests}

The authors declare that they have no competing interests.

\section{References}

1. D. G. Said, H. S. Dua. Chemical burns acid or alkali, what's the difference?[J]. Eye (Lond). 2020; 34(8): 1299-1300.

2. J. P. Craig, K. K. Nichols, E. K. Akpeket al. TFOS DEWS II Definition and Classification Report[J]. Ocul Surf. 2017; 15(3): 276-283.

3. S. Wang, H. Zhang. Upregulation of the IL-33/ST2 pathway in dry eye[J]. Mol Vis. 2019; 25: 583-592.

4. C. Yu, P. Chen, J. Xuet al. hADSCs derived extracellular vesicles inhibit NLRP3inflammasome activation and dry eye[J]. Sci Rep. 2020; 10(1): 14521.

5. F. Bian, F. S. Pelegrino, S. C. Pflugfelderet al. Desiccating Stress-Induced MMP Production and Activity Worsens Wound Healing in Alkali-Burned Corneas[J]. Invest Ophthalmol Vis Sci. 2015; 56(8): 4908-4918.

6. F. Sallusto, M. Baggiolini. Chemokines and leukocyte traffic[J]. Nat Immunol. 2008; 9(9): 949-952.

7. Lu Yang, Zhang Min, Zhou Zhijun. Investigation on the application of GBZ/T 229.2-2010classification of occupational hazards at workplaces Part 2: occupational exposure to chemicals[J]. Chinese Journal of Industrial Hygiene and Occupational Diseases. 2017; 35(11): 837-839.

8. R. Fish, R. S. Davidson. Management of ocular thermal and chemical injuries, including amniotic membrane therapy[J]. Curr Opin Ophthalmol. 2010; 21(4): 317-321.

9. A. Lin, N. Patel, D. Yooet al. Management of ocular conditions in the burn unit: thermal and chemical burns and Stevens-Johnson syndrome/toxic epidermal necrolysis[J]. J Burn Care Res. 2011; 32(5): 547-560.

10. Hosoon Choi, Casie Phillips, Joo Youn Ohet al. Comprehensive Modeling of Corneal Alkali Injury in the Rat Eye[J]. Current Eye Research. 2017; 42(10): 1348-1357.

11. M. D. Wagoner. Chemical injuries of the eye: current concepts in pathophysiology and therapy[J]. Surv Ophthalmol. 1997; 41(4): 275-313.

12. H. Westekemper, F. C. Figueiredo, W. F. Siahet al. Clinical outcomes of amniotic membrane transplantation in the management of acute ocular chemical injury[J]. Br J Ophthalmol. 2017; 101(2): 103-107.

13. B. M. Achauer, S. R. Adair. Acute and reconstructive management of the burned eyelid[J]. Clin Plast Surg. 2000; 27(1): 87-96. 
14. A. Enriquez-de-Salamanca, E. Castellanos, M. E. Sternet al. Tear cytokine and chemokine analysis and clinical correlations in evaporative-type dry eye disease[J]. Mol Vis. 2010; 16: 862-873.

15. S. Nakamura, M. Shibuya, Y. Saitoet al. Protective effect of D-beta-hydroxybutyrate on corneal epithelia in dry eye conditions through suppression of apoptosis[J]. Invest Ophthalmol Vis Sci. 2003; 44(11): 4682-4688.

16. F. Bian, Y. Xiao, M. Zaheeret al. Inhibition of NLRP3 Inflammasome Pathway by Butyrate Improves Corneal Wound Healing in Corneal Alkali Burn[J]. Int J Mol Sci. 2017; 18(3)

17. F. Bian, C. S. Shin, C. Wanget al. Dexamethasone Drug Eluting Nanowafers Control Inflammation in Alkali-Burned Corneas Associated With Dry Eye[J]. Invest Ophthalmol Vis Sci. 2016; 57(7): 32223230.

18. K. S. Na, J. W. Mok, J. Y. Kimet al. Correlations between tear cytokines, chemokines, and soluble receptors and clinical severity of dry eye disease[J]. Invest Ophthalmol Vis Sci. 2012; 53(9): 54435450 .

19. T. Wang, W. Li, H. Chenget al. The Important Role of the Chemokine Axis CCR7-CCL19 and CCR7CCL21 in the Pathophysiology of the Immuno-inflammatory Response in Dry Eye Disease[J]. Ocul Immunol Inflamm. 2021; 29(2): 266-277.

20. A. Gulati, M. Sacchetti, S. Boniniet al. Chemokine receptor CCR5 expression in conjunctival epithelium of patients with dry eye syndrome[J]. Arch Ophthalmol. 2006; 124(5): 710-716.

21. J. E. Turner, O. M. Steinmetz, R. A. Stahlet al. Targeting of Th1-associated chemokine receptors CXCR3 and CCR5 as therapeutic strategy for inflammatory diseases[J]. Mini Rev Med Chem. 2007; 7(11): 1089-1096.

22. R. A. Sack, L. Conradi, D. Krumholzet al. Membrane array characterization of 80 chemokines, cytokines, and growth factors in open- and closed-eye tears: angiogenin and other defense system constituents[J]. Invest Ophthalmol Vis Sci. 2005; 46(4): 1228-1238.

23. W. Choi, Z. Li, H. J. Ohet al. Expression of CCR5 and its ligands CCL3, -4 , and -5 in the tear film and ocular surface of patients with dry eye disease[J]. Curr Eye Res. 2012; 37(1): 12-17.

24. Zhengri Li, Won Choi, Han-Jin Ohet al. Effectiveness of Topical Infliximab in a Mouse Model of Experimental Dry Eye[J]. Cornea. 2012; 31(11 Suppl.): S25-S31.

25. Y. Li, R. Jin, L. Liet al. Therapeutic Effect of Topical Adiponectin-Derived Short Peptides Compared with Globular Adiponectin in Experimental Dry Eye and Alkali Burn[J]. J Ocul Pharmacol Ther. 2020; 36(2): 88-96.

26. T. G. Coursey, N. B. Gandhi, E. A. Volpeet al. Chemokine receptors CCR6 and CXCR3 are necessary for CD4(+) T cell mediated ocular surface disease in experimental dry eye disease[J]. PLoS One. 2013; 8(11): e78508.

27. G. Colucci, F. Invernizzi, Renteria S. Ucedaet al. The CCR5 and CXCR3 Pathways in Hepatitis C Virus Liver Transplanted Recipients Treated by a Direct Antiviral Agent Regimen: Informative Kinetics Profiles[J]. Viral Immunol. 2021; 34(8): 542-551. 


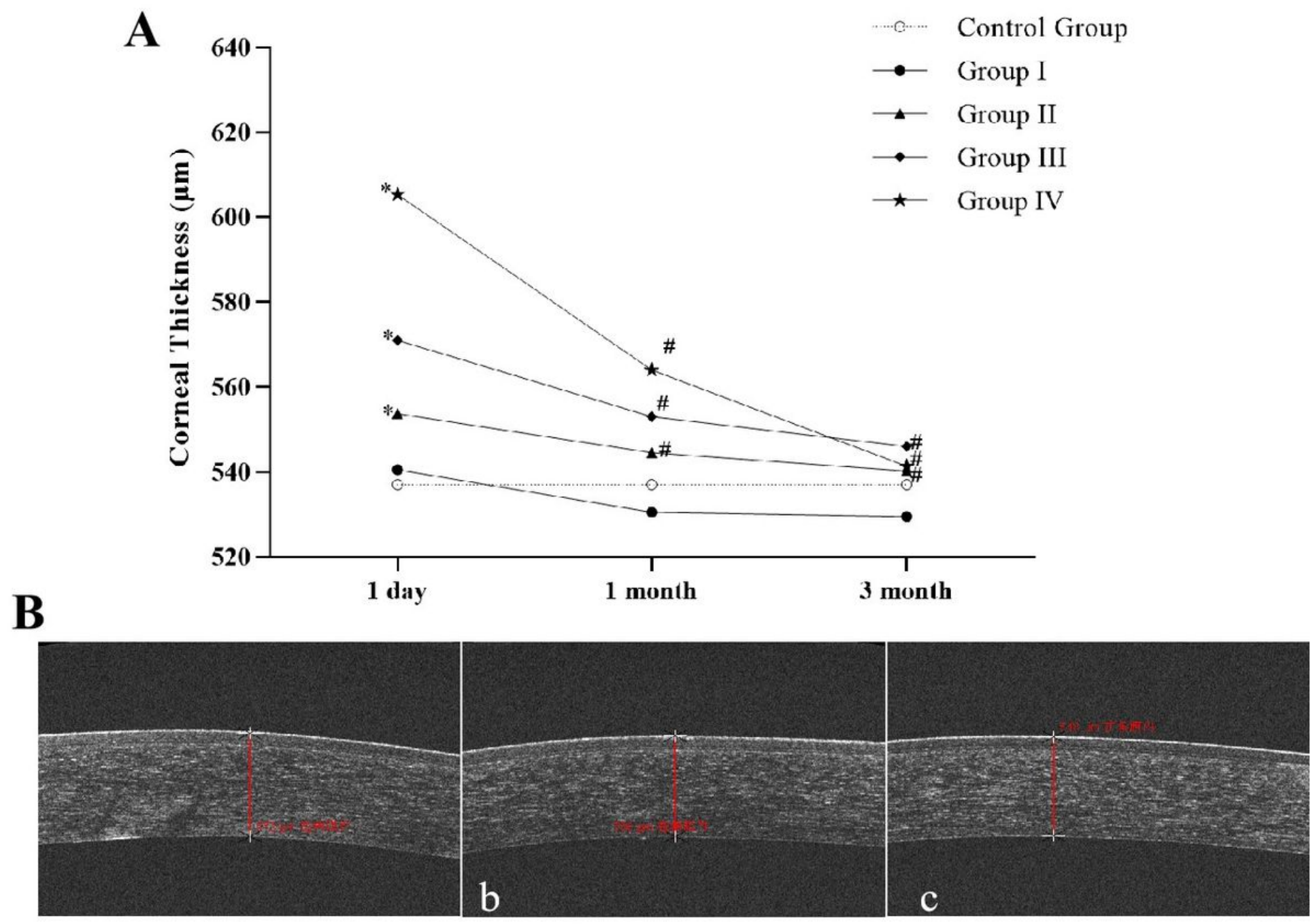

Figure 1

A: Comparison of corneal thickness at 1 day, 1 month and 3 months after injury in each group. (Compared with the control group, ${ }^{*}<<0.05$; Compared with 1 day after injury in the same group, $\# p<0.05$ ) B: OCT image of corneal thickness of a patient with ocular acid burn in group IX. (a. 1 day after injury; b. 1 month after injury; c. 3 months after injury.) 


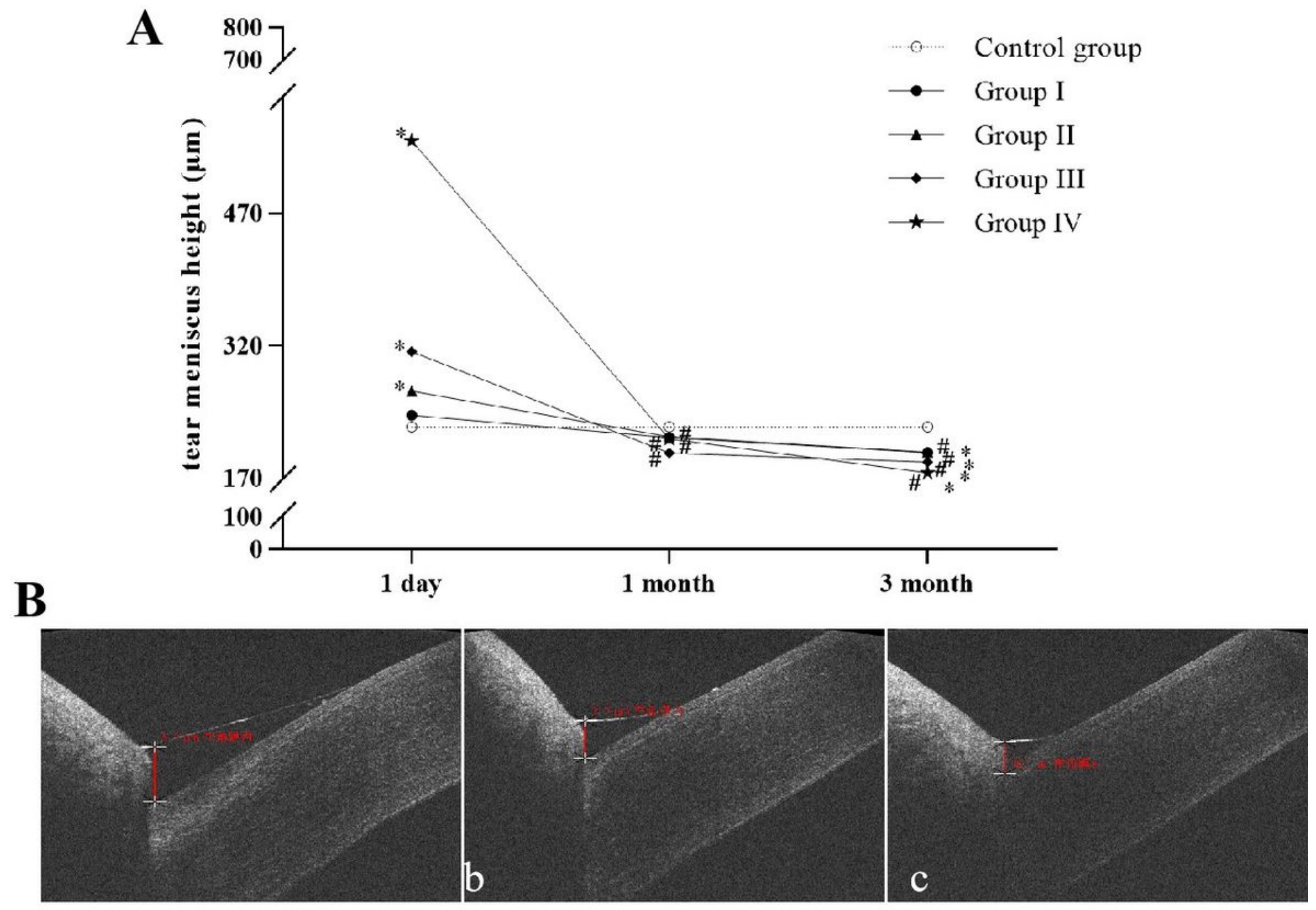

Figure 2

A: Comparison of tear meniscus height (TMH) at 1 day, 1 month and 3 months after injury in each group. (Compared with the control group, ${ }^{*}<<0.05$; Compared with 1 day after injury in the same group, \#p<0.05)

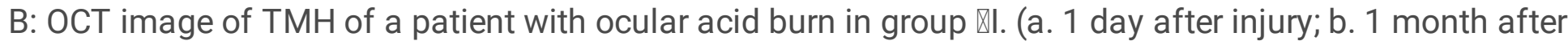
injury; c. 3 months after injury.) 


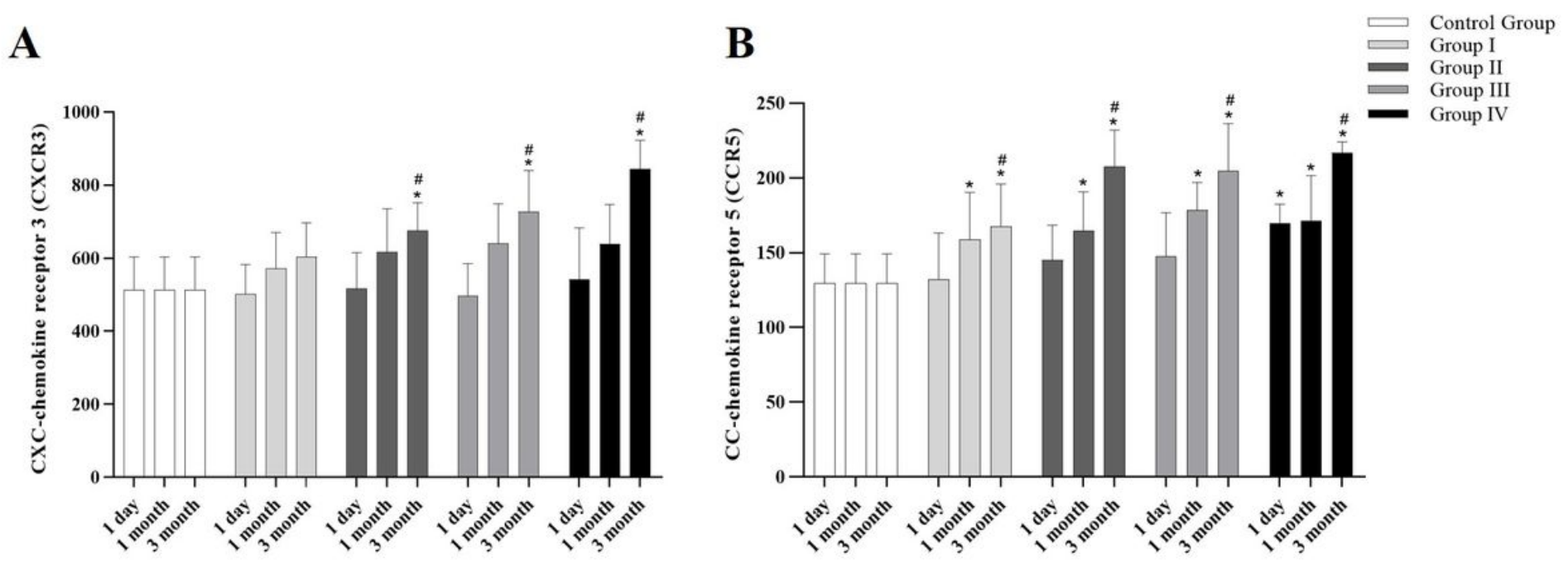

Figure 3

Comparison of CXCR3 and CCR5 protein expression in tears of patients with ocular acid burn in each group 1 day, 1 month and 3 months after injury. A. Comparison of CXCR3 expressions in each group at a different time after injury. Compared with the control group, CXCR3 protein level in group II-IV significantly increased 3 months after injury (all $p<0.05$ ). When compared with 1 day after injury in the same group, the protein levels of CXCR3 in the II-IV group significantly increased 3 months after injury (all p $<0.05)$. B. Comparison of CCR5 expressions in each group at a different time after injury. Compared with the control group, CCR5 protein level in group I-IV significantly increased 1 month and 3 months after injury (all $p<$ 0.05), and the CCR5 protein level in group I was also significantly increased 1 day after injury $(p<0.05)$. When compared with 1 day after injury in the same group, the protein levels of CCR5 in the IIV group significantly increased 3 months after injury (all $p<0.05$ ). 

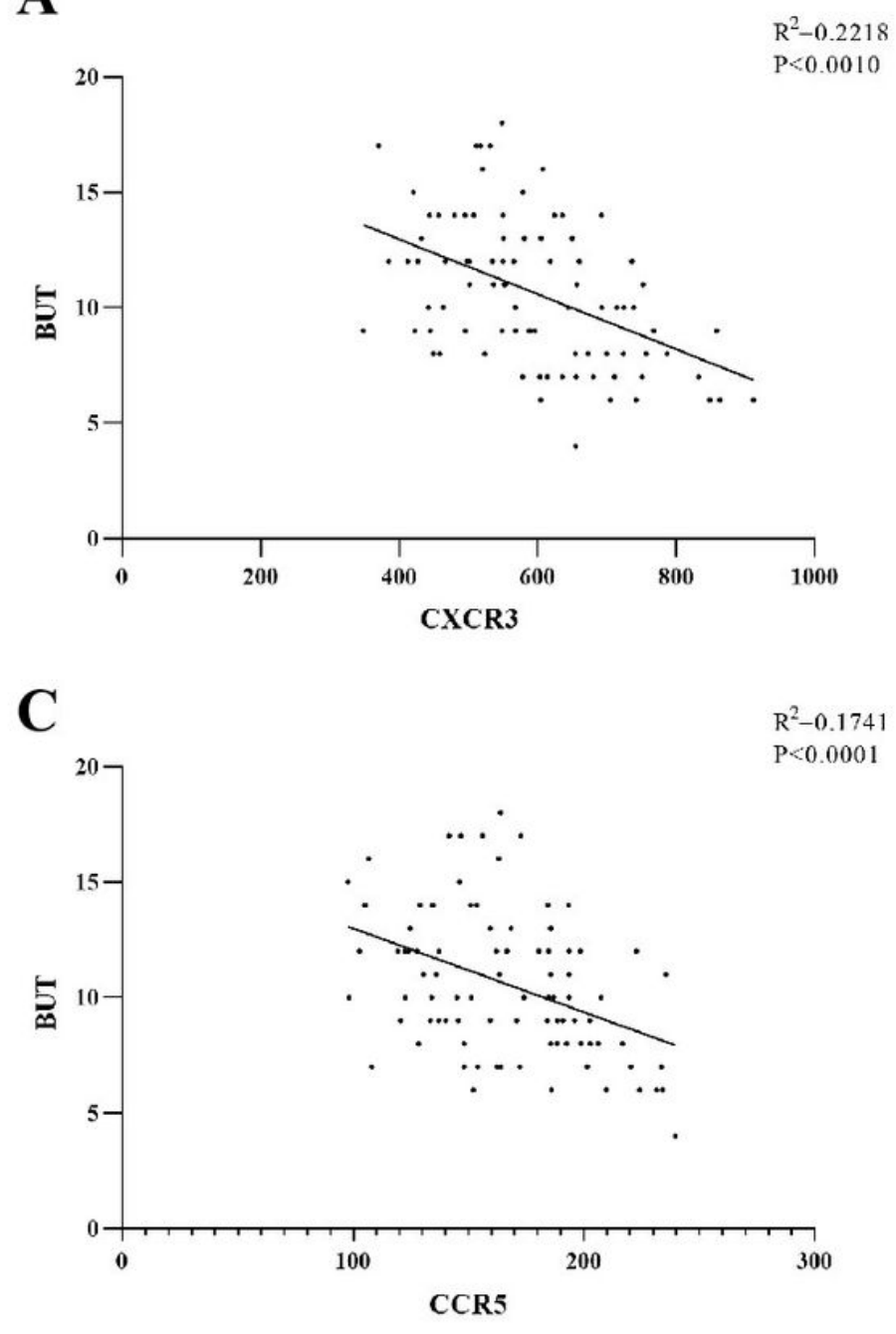

B
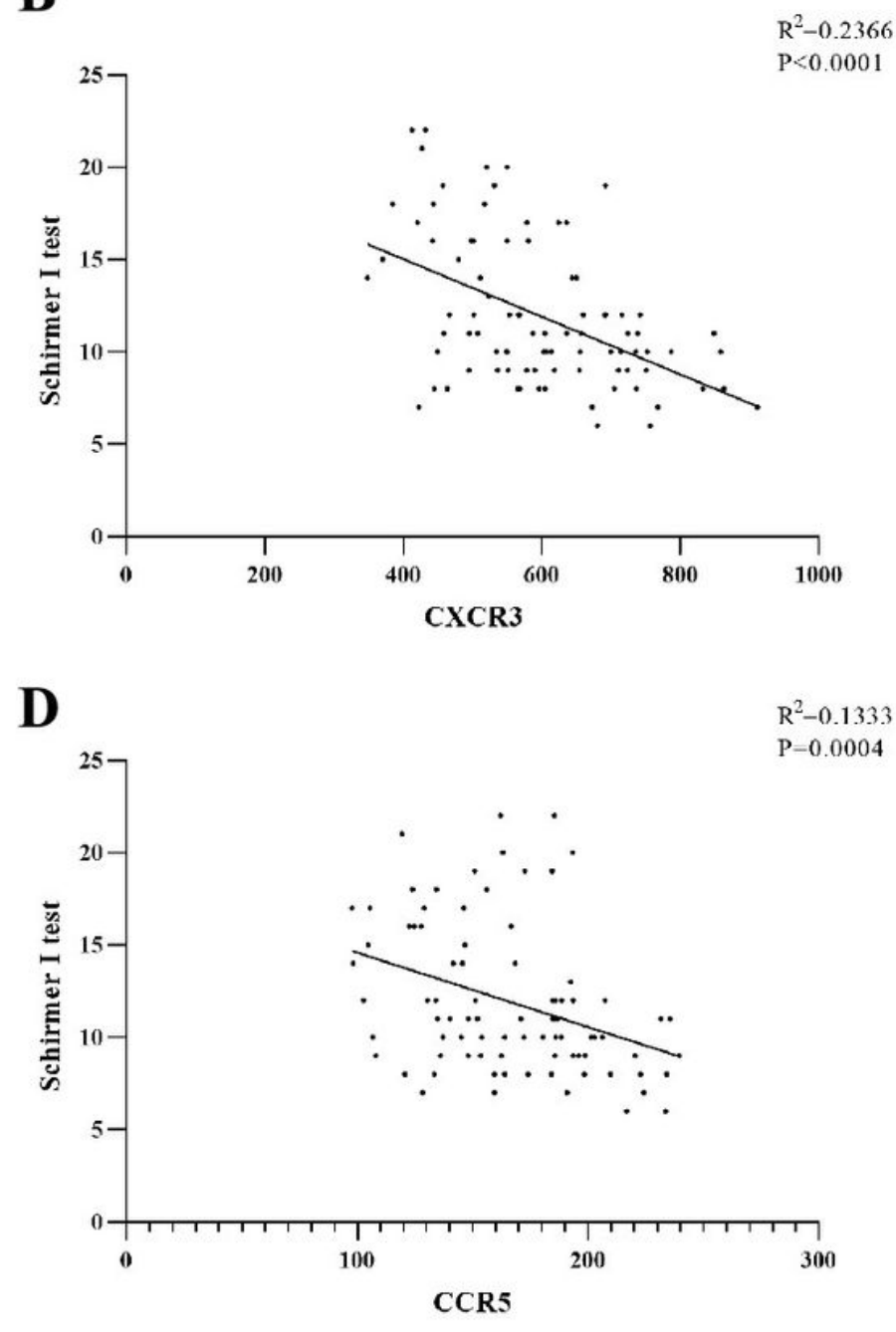

\section{Figure 4}

Correlation between CXCR3 and CCR5 protein expressions and biological parameters. The expression of CXCR3 was significantly negatively correlated with BUT ( $R=-0.4710, P<0.0001)$, and CXCR3 was also significantly negatively correlated with Schirmer I test $(R=-0.4864, P<0.0001)$. The expression of CCR5 was significantly negatively correlated with BUT $(R=-0.4173, P<0.0001), C C R 5$ was also significantly negatively correlated with Schirmer I test $(R=-0.3650, P=0.0004)$. 